\title{
What, if anything, are colours relative to?
}

\author{
JOHN HYMAN
}

If one chooses, one may replace the expression 'frame of reference' by 'frame of reference of an observer'. However, nothing is added to the theory of relativity by any such linguistic transformation. ${ }^{1}$

1. In one of his famous lectures at CalTech, Richard Feynman made fun of philosophers who are prone to say, 'Oh, it is very simple: Einstein's theory says all is relative!' I cannot remember hearing a philosopher say this-although they may have done in Pasadena in the sixties. But colours have been described as relative to observers, relative to the circumstances in which they are observed, relative to languages and even as relative to 'the human perceptual standpoint' or, less chauvinistically, 'the perceptual point of view'. ${ }^{2}$ As this variety of relativisms suggests, the blanket claim that colours are relative and the blanket denial of this claim are equally unhelpful. We need to ask what, if anything, are colours relative to. This is the first question I shall address. The second is whether the relativity of colours, such as it is, implies that they are less real than shapes or intervals in time.

2. To begin with, it is a familiar truth in physics that we cannot talk sensibly about spatial and temporal quantities in nature except in

${ }^{1}$ R. B. Angel, Relativity: The Theory and its Philosophy (Oxford: Pergamon, 1980), p. 109.

${ }^{2}$ Both phrases are quoted from P. F. Strawson, 'Perception and its objects', in J. Dancy, ed., Perceptual Knowledge (Oxford: OUP, 1988), pp. 109f. Nagel claims, in a similar vein, that how something looks, feels, smells, tastes or sounds is relative to a 'general human perceptual point of view'. The View from Nowhere (Oxford: OUP, 1986), p. 14. Relativist claims about colour are also made in M. A. E. Dummett, 'Common sense and physics', in G. F. Macdonald, ed., Perception and Identity (London: Macmillan, 1979), p. 16; D. Wiggins, Needs, Values, Truth, third edition (Oxford: OUP, 1998), p. 107; B. Williams, Descartes: The Project of Pure Enquiry (Harmondsworth: Penguin, 1978), p. 243; C. McGinn, The Subjective View (Oxford: OUP, 1983), p. 9. 


\section{John Hyman}

the context of a frame of reference, in which they can be measured or observed. For scientific purposes, a frame of reference can be defined as a system of coordinates attached to a body of reference. The system of coordinates may be a set of orthogonal Cartesian axes or a different system. But every system of coordinates is a purely conceptual structure and therefore, as Einstein put it, a free invention of the human mind. By contrast, a body of reference is a physical object, which serves as a conventional standard of restsuch as the earth, the sun, or the 'fixed' stars. In combination, the system of coordinates and the body of reference provide a numerical name or label for each position where an object can be located. So measuring velocity, for example, requires a coordinate system attached to a body of reference and a set of instruments to take the measurements with, such as a clock and a ruler, or a radar. ${ }^{3}$

Now if we compare the task of identifying an object's colour, what do we find? First, the role of the coordinate system is played by the system of concepts that is used to identify a colour, e.g. by stating its name. This system of concepts may only distinguish a dozen or so gross colours: red, blue, green, yellow, black, white, etc. Or it may be considerably more sophisticated, like the colour systems invented by Philipp Otto Runge and James Clerk Maxwell, which are among the ancestors of the standard systems used in the printing and manufacturing industries today. ${ }^{4}$ Next, the relative motion of the observer and the object is generally ignoredalthough light from the distant stars is shifted towards red, because light-waves are lengthened when the source recedes from the observer. Finally, we do not use instruments analogous to clocks and rulers to detect gross colours, because our system of gross colour concepts has evolved for use by trichromatic human beings without the aid of samples. But charts are used to identify specific shades, and a colour chart is used as a standard for comparison, which is how a ruler is used too: the ruler provides a sample of a specific length, like the sample of each shade of colour on a chart.

Let us take this comparison a step further. As I have said, we cannot talk sensibly about spatial and temporal quantities, except in the context of a frame of reference. But it does not follow that these

${ }^{3}$ However, we are able to make the simplest spatial and temporal judgements-e.g. about which of two remembered events occurred before the other-without using a ruler or a clock. I return to this point below.

${ }^{4}$ On Runge, see R. G. Kuehni, Colour Space and its Divisions (Hoboken, N. J.: John Wiley, 2003), pp. 59ff. On Maxwell, see P. M. Harman, The Natural Philosophy of Fames Clerk Maxwell (Cambridge: CUP, 2001), ch. 2. 
quantities are relative to a frame of reference, for two reasons. First, there may be a privileged frame, which can serve as the measure of reality and truth-for example, a frame in which the 'fixed' stars are at rest. Accordingly, Earth may not appear to be in motion, but perhaps it truly is in motion, if it is in motion relative to the 'fixed' stars. Secondly, the measurement of a spatial extension or a temporal duration may produce the same result in every frame of reference, whichever body of reference we decide to use. For example, Special Relativity tells us that both the distance in space and the duration of the interval in time between two events are relative to an inertial frame-that is, a frame of reference in which Newton's laws of motion hold true. But it is one of the postulates of Special Relativity that the speed at which light travels through a vacuum is the same in every inertial frame.

Now returning to colours, we find that the same is true. That is, we cannot identify an object's colour except in the context of a frame of reference, but it does not follow that colours are relative to the frame of reference in which they are observed. So let us consider whether in fact they are. Since we generally ignore the relative motion of the observer and the object, the only part of the frame of reference that generally concerns us is the part that is analogous to the coordinate system-that is, our system of colour concepts. The first question about the relativity of colours I shall consider is therefore whether colours are relative to systems of colour concepts. ${ }^{5}$ I shall then consider the relativity of colours to conditions of observation, and finally relativity to observers themselves. I shall not discuss relativity to 'standpoints' or 'points of view', since these terms are very vague, and seem to involve some combination or other of the factors I have mentioned. ${ }^{6}$ For simplicity, I shall

${ }^{5}$ I shall not distinguish between languages and systems of concepts, so I shall treat this question as equivalent to the question of whether colours are relative to colour vocabularies, or sets of intertranslatable colour vocabularies.

${ }^{6}$ The most prominent recent use in metaphysics of the notion of a point of view is made in Thomas Nagel's book The View from Nowhere (Oxford: OUP, 1986). But Nagel's conception of objectivity involves a serious misunderstanding about the role that frames of reference play in physical theories. A note on this point may be of interest to readers familiar with Nagel's book.

In broad terms, Nagel argues that we can attain a more objective picture of the physical world by transcending the human point of view from which physical objects appear to have colours, by abandoning traditional ideas about space and time, and by moving towards a 'view from nowhere'-a view from no point of view at all. Discarding the idea that physical objects 
confine my remarks to gross colours, except at the points where this can be misleading.

3. Where the relativity of gross colour to a system of gross colour concepts is concerned, the relevant facts are these. The colours most English speakers learn to identify by name, and recognize without the use of samples, are tightly constrained by the physiology of the human visual system. As recently as forty years ago, it was widely assumed by philosophers and anthropologists that colours are have intrinsic colours is part of one step we have taken in this direction, under the guidance of Galileo and Descartes. And since Special Relativity implies that space and time are relative to an inertial frame, discarding shapes is part of another step. Nagel describes Einstein's 'huge step' towards an objective view of the world as follows (p. 76):

What formerly seemed to be an objective conception of absolute space and time was revealed to be a mere appearance, from the perspective of one frame of reference, of a world whose objective description from no frame of reference is not given in a four-dimensional coordinate system of independent spatial and temporal dimensions at all. Instead, objects are objectively located in relativistic space-time, whose division into separate spatial and temporal dimensions depends on one's point of view.

These remarks involve three issues: first, the contrast between Newton's conception of absolute space and absolute time and Einstein's discovery that spatial and temporal intervals are relative to inertial frames; second, the contrast between a description of the world from the perspective of one frame of reference or another and a description from the perspective of no frame of reference at all; and third, the idea expressed in Minkowski's famous remark, 'Henceforth, space by itself, and time by itself, are doomed to fade away into mere shadows...', which I comment on below.

The issue that is immediately relevant to Nagel's notion of objectivity is the second. Special Relativity, he suggests, is an advance towards an objective view of the world, because Newtonian physics describes physical phenomena 'from the perspective of one frame of reference', whereas Special Relativity describes them 'from no frame of reference'. But this could not be further from the truth. The Principle of Special Relativity is indeed that physical laws, including the laws of electrodynamics, are the same in all inertial frames. But it does not follow that Special Relativity enables us to describe the world 'from no frame of reference'. No such description is possible. There is no description of the world in which 'objects are objectively located' from no frame of reference, because without a reference frame we cannot assign spatial or temporal quantities to natural phenomena at all. It is therefore a mistake to think of any physical theory as providing a 'view from nowhere' of the physical world. 
carved out of an undifferentiated visual flux by words, or by habits of mind instilled by learning words. For example, Quine claims that 'whether some arbitrary interval in the spectrum is $a$ colour [e.g. red] ... depends on the casual matter of there being a word for it; and this matter of vocabulary varies from culture to culture. ${ }^{77}$ But the evidence does not support this nominalist view. ${ }^{8}$ On the contrary, our gross colour vision is naturally organized around a set of focal areas, which are centres of variation for each of the gross colours-for example, the range of shades that qualify as paradigmatic examples of the colour red. The boundary lines we draw between gross colours are more variable and fluid. But of course they cannot be located very close to the focal areas.

In effect, our perception of gross colours is a product of the relative salience of some differences in spectral reflectance, by comparison with others. For example, the difference in wavelength between 425 and $475 \mathrm{~nm}$ (nanometres) is the same as the difference between 475 and $525 \mathrm{~nm}$. But if the wavelength of light projected onto a screen is gradually changed from 425 to $475 \mathrm{~nm}$ there will be very little noticeable change in its colour. It will become a slightly brighter shade of blue. Whereas if the light is gradually changed from 475 to $525 \mathrm{~nm}$ it will change markedly, from blue to green. To see why this this happens-why the same kind of change in the light corresponds to a very different kind of change in the colour we perceive-consider a single photoreceptor. If we plot its spectral sensitivity, we get a bell-curve. The top of the curve is where the photoreceptor's response peaks; and the sides are where it tails off. Now it should be obvious that the same difference in wavelength will correspond to a greater difference in response on the two steep sides of the curve than on the top. Of course, the mechanisms which explain why our sensitivity to a fixed difference in wavelength varies along the visible spectrum are complex. And besides, several of the gross colours we perceive are not spectral colours. Nevertheless, the simple model shows how a stimulus varying at a constant rate can elicit an abrupt change in response even in a simple mechanism, where the 'casual matter' of vocabulary does not arise.

${ }^{7}$ W. V. O. Quine, The Roots of Reference (La Salle, Ill.: Open Court, 1973), p. 71.

${ }^{8}$ The pioneering work was published in B. Berlin \& P. Kay, Basic Color Terms: their universality and evolution (Berkeley, CA: University of California Press, 1969). The basic colour terms they identify are: black, white, red, green, yellow, blue, brown, purple, pink, orange and grey. Some aspects of their work are still controversial; but the simple point that basic colours are not linguistic artefacts is not. 


\section{John Hyman}

The focal areas at the heart of gross colours and the boundary areas that separate them are, then, the places where we are relatively insensitive and relatively sensitive respectively to similar differences in reflectance. However, other vertebrates have colour vision, and so do butterflies and bees; and colour vision differs from one species to another. In the first place, the photoreceptors of birds and bees are sensitive to lower wavelengths of light than human photoreceptors are. Secondly, normal human colour vision is trichromatic: it depends on three kinds of photoreceptors, each containing a photopigment with a characteristic spectral response curve. Ungulates, such as pigs, sheep and horses are dichromatic; while birds, and some fish and amphibians, are tetrachromatic. Thirdly, the boundaries we draw between gross colours are a complex function of the spectral response curves of our photoreceptors. So trichromatic animals whose photoreceptors have different spectral response curves from ours will perceive these boundaries in different places from us, and will no more be able to see the boundaries we perceive than we are able to see theirs.

Hence, the set of gross colours that English-speakers learn to name is anthropocentric, in the sense that it reflects the colour foci and the approximate boundaries between gross colours that most trichromatic human beings perceive. It is not specifically Anglocentric, or less so than Quine seems to have thought. But it is anthropocentric, in a deeper sense than the imperial system of measurements is-with its inches, feet and yards. For if two objects have the same dimensions in the imperial system, they have the same dimensions in the metric system, or in a system based on the body of a cat. But two objects that are both red or both green need not have the same gross colour from a set that is tailored to fit the visual system of another species.

4. In order to decide whether gross colours are relative to systems of colour concepts, imagine that the gross colour of an object is identified with our familiar anthropocentric system of gross colour concepts, and also with a system devised for a dichromatic species, such as the horse. We shall ignore questions about the conditions of observation and questions about the colour experiences or behaviour of observers. For the moment, we are only interested in the alleged relativity of gross colours to systems of colour concepts.

Now imagine that a grassy bank is found to be green when the anthropocentric system is being used, and that it is found to be a colour which encompasses some shades of red, some shades of 


\section{What, if anything, are colours relative to?}

green and some shades of brown when an equine system is used. It may seem as if we could argue as follows: 'We have seen that there can be two reasons to deny that a spatial or temporal quantity is relative to a frame of reference. First, there may be a privileged frame of reference, such as a frame in which the "fixed" stars are at rest; and secondly, measurement may produce the same result in every frame of reference, as it does in the case of the velocity of light. Presumably, the same is true of a quality, such as gross colour. But in this case "measurement" has produced two different results: first green, and then an equine gross colour, for which we have no name. And there is no reason, apart from chauvinism, to prefer the human system of colour concepts. Hence, gross colour is relative: it varies from one system of colour concepts to another.'

If this argument were sound, we should have to say that nothing is green or red without qualification, but only relative to a system of colour concepts. But in fact the argument is fallacious. For although we found that the grassy bank has two colours-first green, and then a nameless equine colour-this is not like finding that two measurements of an object's velocity have yielded inconsistent results. It is like finding that an object is located in two overlapping regions. Consider an analogy. Barnsley, the South Yorkshire town, is in the Catholic Diocese of Hallam and in the Anglican Diocese of Sheffield. These are two different regions which overlap. So one and the same town can be in both. Hence, we should not say: Barnsley is inside the Diocese of Hallam relative to the Catholic Church, but not relative to the Anglican Church. We should say without qualification that it is inside the Diocese of Hallam, and add if we wish that Hallam is a Catholic diocese. Similarly, we should not say that the colour of the grassy bank is green relative to the anthropocentric system of gross colours, but not relative to the equine system. We should say that it is green without qualification, and add if we wish that green is an anthropocentric colour.

If we return to the idea of a frame of reference, we shall see why we have obtained this result. We saw that a frame of reference consists of a system of coordinates and a body of reference; and that the system of coordinates is a conceptual construct, whereas the body of reference is a physical object. When a physical quantity is said to be relative to a frame of reference, this does not mean that it varies depending on the system of coordinates that is used to measure it. It means that it varies depending on the body of reference that is used as a standard of rest. For example, according to Special Relativity, if the length of a rod is one metre in an inertial frame in which it is at rest, then its length will be less than 


\section{John Hyman}

one metre in an inertial frame in which it is in motion. ${ }^{9}$ But the length of the rod will not differ depending on whether the coordinate system is set of orthogonal Cartesian axes, an oblique Cartesian system, in which the axes are rectilinear but not orthogonal, or a system in which the axes are curves. As Wittgenstein laconically observed, by a new notation no facts of geography are changed.

Admittedly, the anthropocentric system of gross colour concepts is not a coordinate system. If we use it to identify an object's colour, this is more like locating a town in a diocese than like giving coordinates in longitude and latitude or an Ordnance Survey grid reference. However, the role of the system of concepts in a frame of reference is essentially the same, whether it is mathematical or not. Gross colours are therefore not relative to systems of colour concepts.

5. I shall turn now from the reference frame to the conditions in which an object's colour is observed. The questions here are whether colours are relative to illumination; to the extent and quality of the atmosphere between an object and an observer; or to the background against which an object is seen. For example, suppose that the colour of a mountain appears to change from white at midday to pink at dusk; that a distant range of hills looks bluish, by comparison with one that is closer; or that a yellow pigment looks more saturated on a blue ground. Should we say that the colours of these things are relative? For example, should we say that the mountain is white at midday, and that it is pink at dusk? Or should we deny these things, and privilege one kind of illumination, one distance and one kind of ground? Should we say that the mountain looks pink at dusk, but that broad daylight reveals its true colour; and that proximity reveals the true colour of the hills?

I shall begin by making two preliminary observations. First, we cannot deny that an object's colour is relative to the conditions in which it is observed unless we can distinguish between the colour it looks in various conditions and the colour it is. And we cannot draw this distinction unless we can define the conditions in which the colour it looks $i$ s the colour it is. A blithe mention of 'standard conditions' achieves nothing. For the question is whether there really are such standard conditions; and if so, what they are. Secondly, we cannot define the conditions in which the colour an object looks is the colour it is unless one system of colour concepts or another is in play. This need not be our familiar system of gross colour con-

${ }^{9}$ Except that there is no contraction orthogonal to the direction of its motion. 
cepts-red, green, blue, yellow, etc. But 'the colour it is' is just an empty phrase, unless a range of colours has been defined. It is tempting to point at an object and imagine that 'the colour it is' simply refers to THIS. But the question of what colour something is is a question about the identity of its colour. And as Wittgenstein pointed out, one does not define a criterion of identity by emphatic stressing of the word 'this'.

Now as a matter of fact, in many cases, although not by any means in all, we follow the principle that the gross colours of objects are the gross colours they appear to have in daylight. Of course, our colour perception is responsive to subtle changes in the intensity and colour temperature of light, to its direction and the degree to which it is diffused. But each of our gross colours encompasses so many discriminable shades that these subtle changes do not generally affect the gross colours we perceive. In other words, the loose definition of 'standard conditions' we implicitly accept corresponds to the broad range of shades of colour each gross colour includes. But there are many anomalous cases. That is to say, there are many cases in which such a principle cannot easily, or cannot possibly, be applied. And so there are many cases in which the distinction between the gross colour an object looks in varying conditions of illumination and the gross colour it is is difficult or impossible to sustain.

For example, J.L. Austin asks:

What is the real colour of the sky? Of the sun? Of the moon? ...

We say that the sun in the evening sometimes looks red-well, what colour is it really? (What are the 'conditions of standard illumination' for the sun? $)^{10}$

As this passage suggests, we often confine ourselves to the question of what colour an object looks in one set of conditions or another, and discard the question of its real colour. And for ordinary purposes, it does not matter whether the idioms we choose appear to relativize the colour a thing is or the colour it looks-for example, whether we say that the sun in the evening sometimes is red or that it sometimes looks red; or whether we say that the hills become bluish at a distance or that they look bluish at a distance. It does not matter which of these things we say because the distinction between being a certain colour and looking a certain colour is precisely the

${ }^{10}$ J. L. Austin, Sense and Sensibilia (Oxford: OUP, 1962), p. 66. C. L. Hardin, Colour for Philosophers (Indianapolis, Ind.: Hackett, 1988), pp. $67 \mathrm{ff}$.) discusses several other less familiar cases where the idea of standard conditions is inapplicable. 


\section{John Hyman}

distinction we no longer make in this kind of case. But the fact that we do not or cannot make this distinction in some cases does not imply that we do not or cannot make it in any cases. And the fact that the daylight principle cannot be used to fix the colour of every visible object does not imply that it cannot be used to fix the gross colour of a broad range of visible objects; or that it is a kind of fiction, which misrepresents the facts.

In general, then, we tend to be absolutists about gross colourthat is, we tend to assign specific gross colours to objects without relativizing these colours to the conditions in which they are observed-first, when standard conditions can be easily defined and, once defined, identified from one occasion to another; and secondly, when we are more interested in the relatively stable properties of visible objects than in their variable relationship with light. But our conceptual habits tend to be as relaxed and fluid as the case allows, and in this case the latitude is large. So the question of whether gross colours are relative to the conditions in which they are observed does not have the same answer, or even a definite answer, in every case.

Three further points should be noted. First, I have been talking about the relativity of the gross colours of objects, in conformity with the principle that we cannot raise the question of whether an object's colour is relative to the conditions in which it is seen unless one system of colour concepts or another is in play. What I have said about gross colours does not automatically apply to finer shades of colour, because finer shades of colour are less stable than gross colours in varying illumination, in the way that a small boat is less stable than a big one in rough weather. Daylight or 'clear grey'-a phrase Cezanne used to describe the kind of weather that he wanted for a sitting-is a sufficiently precise definition of the standard illumination for the gross colours of what Austin liked to call moderate-sized dry goods. But colour systems that distinguish several thousand hues are commonly used in conjunction with a more precisely defined standard light-source-for example, a tungstenfilament lamp with a specific colour temperature.

Secondly, as we approach the limit of barely discriminable shades, the definition of a shared system of named or numerically labelled shades becomes less feasible, because individual differences make it more difficult for us to agree on the correct use of a standard chart. At the limit, where no system of colour concepts is in use, we can still decide whether two colour samples are indistinguishable by a given observer in a given light; but the question of what colour a sample is simply cannot be raised if there 
is no system of colour concepts in play. Hence metamers-that is, matching samples with different reflectances-are relative to observers and conditions of observation. But it is a mistake to infer that the same is true of gross colours. This inference ignores the cardinal principle that specific colours cannot be identified, except by means of specific colour concepts.

Finally, when we cannot distinguish straightforwardly between the coloured object and the illumination, we have no option but to say that the object's colour changes with the light. For example, the sky may be uniformly blue at midday and streaked with red and pink at dusk. It makes scant sense to ask which of these is its true colour, because the colour of the sky $i s$ the colour of sunlight that has passed through the atmosphere's diffusing filter.

6. Are colours are relative to observers? To begin with, our perceptions of objects obviously depend both on their nature and our own. For example, if a trichromatic human observer and a horse both see a grassy bank, the gross colours they will see are different: the human observer will normally see the anthropocentric colour green, whereas the horse will normally see a colour which encompasses some shades of red, some shades of green and some shades of brown. And the difference between these gross colours will be due to the difference between the human and the equine visual systems.

However, we should not infer that the gross colour of the grassy bank is green relative to a trichromatic human being but not relative to a horse. For as we saw earlier there is no reason to deny that it has both colours. Indeed, it is tempting to say that in principle an object has as many gross colours as we care to define, as long as it lies within their boundaries. But the point of defining a gross colour is that it can be identified without comparing the object with a sample, and a gerrymandered colour cannot satisfy this requirement. (This is the point Quine failed to notice.) So it is better to say that the class of objects with the same gross colour encompasses a great family of reflectances-which has, as large families do, some degree of indeterminacy at the margins; and that objects with various reflectances may have the same gross colour from an anthropocentric system, while having different gross colours from a system designed for another species.

We should therefore be pluralists about gross colour-that is, we should acknowledge that the gross colours we are familiar with are not the only ones it is possible to perceive. But we should not relativize gross colours to observers. For what varies from one kind 


\section{John Hyman}

of animal to another is not the colour of the grassy bank, but the colour that is normally perceived, when it is seen. It is true that a grassy bank normally looks green to a man but not to a horse; but we should not infer that it is green relative to a man but not a horse. The mistaken idea that we should make this inference stems mainly from forgetting the cardinal principle that we cannot identify an object's colour, except in the context of a frame of reference. For if we ignore the role of the frame of reference, then instead of thinking, correctly, that the grassy bank is green, and that green is a gross colour relative to a trichromatic human being, we produce an elision of this thought in our minds, and think instead that the grassy bank is green relative to a human being.

If we ignore the role of the frame of reference, the idea that colours are relative to observers becomes hard to resist. But once we remember that the frame of reference is indispensible, we should be able to see that far from the colour of an object being relative to observers, observers qualify as such only relative to specific frames of reference. Of course it is obvious in general terms that only some animals qualify as observers of colours, from the mere fact that only some animals are sighted. The less obvious point-although it is surely not much less obvious-is that which animals qualify as observers depends on the frame of reference that is being used. Naturally, a normal trichromatic human being qualifies as an observer relative to the anthropocentric system of gross colours, because the system evolved to fit his grip. In general, if we assume, for simplicity, that the gross colour of an object is to be identified by unaided observation, an animal will qualify as an observer in the context of a given frame of reference if, and only if, the gross colours which the system provides for are the gross colours which that animal perceives. ${ }^{11}$ For the anthropocentric system, standard tests for colour vision can be used to decide which people qualify and which do not.

In a sense, we can therefore say that there is a preestablished harmony between qualified human observers and the anthropocentric reference frame in which gross colours are observed. The mistake is to confuse this with a preestablished harmony between qualified observers and the facts.

7. That there is this latter kind of preestablished harmony is the substance of Democritus's famous remark: 'Colours, sweetness,

${ }^{11}$ The assumption is necessary because a suitably designed colour chart will enable a trichromat to assign gross colours belonging to a dichromatic system, although not the reverse. 
bitterness, exist by convention: in reality, there are atoms and the void.' This remark invokes the antithesis between nomos (law, convention, custom) and phusis (nature), which occupied a central place in philosophical and political debate in Athens in the fifth century $\mathrm{BC} .{ }^{12}$ But we do not need to understand the intellectual context of the remark in order to perceive its force. For we are not infallible judges of physical reality; and it must therefore be possible to distinguish between the properties we are severally or jointly willing to predicate of a physical object and the properties it really does possess. In effect, Democritus is claiming that this condition is not satisfied in the case of colours and tastes, and many philosophers have agreed. For example, Hume writes:

The appearance of objects in daylight, to the eye of a man in health, is denominated their true and real colour, even while colour is allowed to be merely a phantasm of the senses. ${ }^{13}$

More recently, Bernard Williams has expressed essentially the same idea, although he follows Democritus in referring to agreement between observers, where Hume refers to the qualified observer in general. 'Our distinctions between what seems green and what is green' Williams writes, 'are essentially based on agreement within the range of human experience'. ${ }^{14}$ And P. F. Strawson claims, in a

${ }^{12}$ E. R. Dodds suggests that the antithesis was first explicitly discussed in the field of medicine: 'are health and sickness determined mainly by a man's phusis, his "constitution" as we say, or mainly by his nomos, i.e. his customary regime of diet, exercise, and so forth? But very soon the problem assumed an ampler scope. When law and human nature conflict, which ought we to follow? Is the social restraint which law imposes on nature a good or a bad thing? For the Sophists that was the grand question.' E.R. Dodds, 'The Sophistic movement and the failure of Greek liberalism', in The Ancient Concept of Progress and other essays (Oxford: OUP, 1973), p. 99.

${ }^{13}$ D. Hume, 'Of the standard of taste', in Essays Moral, Political and Literary, p. 234.

${ }^{14}$ Descartes: the project of pure enquiry, p. 242. Williams also describes the term 'green' as 'relative, relating to human tastes and interests' (p. 243), and says that concepts of secondary qualities are 'peculiarly relative to our experience' (p. 244). For the full argument, see Williams, op. cit., pp. 240246. My general impression of this complex argument is that Williams fails to distinguish with sufficient care between the question of which truths are 'peculiarly relative to our experience' and which concepts are anthropocentric. This leads him to infer from the anthropocentricity of our system of gross colour concepts, to which he rightly draws attention, that objects do not "'really" have one colour rather than another'. 


\section{John Hyman}

similar vein, that where the ascription of colours is concerned, 'the standard of correctness [is] intersubjective agreement. ${ }^{15}$ If this is true, our agreement that something is green-at least if it is reached in favourable circumstances-is sufficient to guarantee that it is green. And this dependency of colours on agreement implies that they are not properties that objects really possess. ${ }^{16}$

I shall criticize this doctrine in a moment; but before I do so I shall make a few preliminary remarks and note a point of qualified agreement with Williams's view. It would clearly be a mistake to say that what is right is never fixed by agreement. For it is, in the case of social norms. For example, the right way to pronounce a word is the way it is generally pronounced by native speakers and in some societies the right way to express appreciation at the end of a musical performance is to clap. Thus we can say about rules of pronunciation, and about various forms of polite behaviour, that the right way of doing something is the way that is agreed on in the relevant group. We can even say that the esse of a social norm is percipi, as long as percipi means being acknowledged and not being observed. But-this is the point on which I agree with Williams and many others-if agreement fixes the truth about something, the truth it fixes is essentially about ourselves. Or, to put the same point in a different way, agreements are the only realities that agreement can produce.

It is self-evident that conventions exist by convention; but it is not self-evident that colours do as well. If we say that they do, we are not bound to hold that the case of colours and the case of politeness are exactly alike. ${ }^{17}$ But we are bound to hold that if trichromatic observers agree in favourable circumstances that something is green, then it follows, as a matter of logic, that it is.

15 P. F. Strawson, 'Perception and its objects', repr. in Perceptual Knowledge, ed. J. Dancy (Oxford: OUP, 1988), p. 112.

${ }_{16}$ The thought that agreement provides this guarantee can also be founded on a general commitment to the notion that truth cannot outstrip best opinion. There are compelling reasons to reject this idealistic doctrine; but they are not relevant here, because if the doctrine were true it would have no special application in the case of colours. On this topic, see W. Künne, Conceptions of Truth (Oxford: OUP, 2003), ch. 7.

${ }^{17}$ We can still maintain that human nature leaves us with a freer hand to vary social norms, and individual responses are correspondingly determined to a greater degree by custom-although human nature does not leave us a totally free hand. Rules of pronunciation are constrained by the shape of the human mouth and tongue, and norms of politeness by natural reactions of disgust. For example, it could not be a conventional expression of gratitude for a meal to vomit on one's host. 
8. The claim that agreement fixes the truth about an object's colour and the claim that colours are relative to communities of observers are in essence one and the same. Hence, we should expect to find that the former claim projects a feature of our system of gross colour concepts onto the coloured objects we perceive, just as the latter has been shown to do. And in fact we do find this. As we have seen, our system of gross colour concepts is essentially based on our shared disposition to find some differences in spectral reflectance more salient than others. But if a system of concepts is essentially based in this way on agreement in experience, it does not follow that the facts it is used to state are too. Crimes are defined by laws; and in a democracy laws are fixed by agreement. But the fact that a man has broken the law is not. If it were, the jury's verdict could not be mistaken, and it would be logically impossible to convict an innocent man. But this is not impossible, however favourable the circumstances are in which he is tried.

The same is true in the case of colours. Our anthropocentric system of gross colour concepts is fixed by agreement, tightly constrained by the nature of our visual system. Hence, in questions of law-e.g. where the approximate boundaries of a gross colour lie-we are authoritative. In fact, this is true in a quite literal sense, since if we include our ancestors among ourselves, we are the authors of the concepts that we use, just as we are the authors of our laws. But we are not authoritative in questions of fact. Like every other kind of judgement that we make, our judgements about what seems green and what is green cannot be tested by a jury of angels: human jurors are the only ones we have. But we cannot bestow colours on objects merely by agreeing that they possess them, however sober and responsible we are. In general, that takes a lick or two of paint.

So, where questions about the colours of physical objects are concerned it is reasonable to accept the judgement of qualified and disinterested observers-as it generally is, in questions of fact. But mistakes are conceivable in the most favourable circumstances; no one's impression is irrefragable; and agreement cannot remove the logical possibility of a mistake. In many cases it is true that the broader the agreement we secure, the more confident we are entitled to be that we are right. But there are no circumstances in which a consensus about an object's colour is logically guaranteed to be the truth-however extensive the consensus is. Of course, we can if we wish define 'favourable circumstances' to mean circumstances in which the consensus of qualified observers is true. But this 


\section{John Hyman}

manoeuvre does not exclude the logical possibility of error: it merely transfers it to the question of whether these circumstances obtain. We can pull the rug across one gap; but the result is that we expose the other.

It does not follow that we should always feel doubtful about our judgements of colour. We should not, always-any more than we should always feel doubtful about our judgements of shape. Nor does it follow that there are no circumstances in which we could not seriously entertain the possibility that we were wrong. No doubt, there are such circumstances-as there are with shapes. But the question here is not about what ought to satisfy us that an object has a certain colour, or even about what ought to absolutely convince us that it does. It is whether there are any circumstances in which the opinion of normal observers-i.e. normal trichromatic human beings-carries a logically impervious guarantee of truth. If we distinguish carefully between questions about our colour concepts and questions about the facts we use them to state, it is clear that there are no such circumstances-regardless of how much agreement is secured.

9. It follows that there are both similarities and differences in the way in which judgments about colour and judgments about space and time are made. On the one hand, as I noted earlier, we are able to make primitive spatial and temporal judgements without using a ruler or a clock, just as we are able to make judgments of gross colour by unaided observation. For example, we can often say which of two past events occurred before the other, or whether two objects are roughly equidistant from a third. These judgments cannot be described as measurements, if the term 'measurement' is reserved for the case where an observation is expressed with actual numbers. But they are evidently judgments about space and time.

Furthermore, rulers and clocks refine our ability to make these kinds of judgments by providing samples of lengths and temporal durations, and colour samples refine our ability to make colour judgments in a similar way. Some philosophers are still inclined to think that we decide on an object's length by an objective technique, viz. measurement, whereas we decide on its colour by appealing to the verdict of the mob. But as Armstrong pointed out long ago, the use of a standard chart to decide on an object's colour is no more reliant on the senses or on agreement than the use of a ruler to decide its length. It is true that we have to see that the object's surface has the same colour as the sample on the chart; but we have 
to see that the object being measured coincides with the points on the ruler. ${ }^{18}$

On the other hand, there is an equally important difference between colours and distances or durations, which Armstrong does not mention. For the measurement of space and time can be cut loose from any reliance on perception, in a way that the observation of an object's colour cannot. For example, a clock is an instrument which ticks out equal intervals of time; and it need not be designed to match an intuitive sense of temporal duration. But a spectrophotometer cannot decide what colour an object is, as opposed to measuring its reflectance, unless it has been calibrated to match the sorting behaviour or the colour judgments of an animal that sees them.

Hence, where colours are concerned, human observers staff the highest court of appeal; whereas questions about temporal duration can be referred to clocks. ${ }^{19}$ This is surely one source of the common intuition that Democritus was right. For it is tempting to say: 'Since there is no implacable machine to overturn the verdict of our senses, we have a free hand! These things are up to us!' But this is a mistake. We might just as well say: 'Since there is no god Khronos to overturn their verdict, how long it takes to boil an egg is up to the clocks!' It is true that agreement among observers leaves us in no doubt that grass is green; and agreement among clocks leaves us in no doubt that eggs are boiled in five minutes at sea level. But the statement that grass is green is not a covert generalization about observers, any more than the statement about eggs is a covert generalization about clocks. ${ }^{20}$

10. I shall turn now from the question of whether colours are relative to concepts, conditions of observation or observers to the question of whether the relativity of colours, such as it is, impugns or diminishes their reality. The most stringent view we can take on this question is that if a property is relative to a frame of reference,

${ }^{18}$ D. Armstrong, Perception and the Physical World (London: Routledge \& Kegan Paul, 1961), p. 182.

${ }^{19}$ I ignore the point that in many states the highest court of appeal will not decide questions of fact.

${ }^{20}$ Precisely this kind of mistake has led some physicists and philosophers to embrace an idealist interpretation of Special Relativity. They have mistakenly supposed that the basis of the theory does consist in hypotheses about the behaviour of clocks and measuring rods. There is an excellent discussion of such an interpretation (namely, Eddington's) in R. B. Angel, op. cit., pp. 104ff. 


\section{John Hyman}

we are immediately bound to exclude it from a scientific conception of the real world. This view was famously expressed by Minkowski, in the 1908 paper in which Special Relativity was given its fourdimensional formulation:

Henceforth, space by itself, and time by itself, are doomed to fade away into mere shadows, and only a kind of union of the two will preserve an independent reality. ${ }^{21}$

More prosaically, although Special Relativity relativizes the spatial and temporal intervals between two events to an inertial frame, there is a function of these two quantities, called the 'space-time interval', which is the same in every inertial frame.

However, despite Minkowski's poetic remark, Special Relativity does not imply that space or time is an illusion. If we imagine that it does, we are confusing the distinction between absolute and relative and the distinction between reality and illusion. For example, Special Relativity does indeed imply that simultaneity is relative to an inertial frame; but far from implying that simultaneity is an illusion, it provides a well-defined method for deciding whether two events are simultaneous. Some philosophers have argued that time is an illusion: that no man is really born before his children, and that no battle really begins before it ends. But whatever can be said in favour of this doctrine, Special Relativity does not imply that it is true.

A more moderate view is that if a property is found to be relative to a system of concepts or to observers, we can no longer hold that it is real. I accept this view, simply because it is obvious, as I have said already, that we are not, even collectively and even in the most favourable circumstances, infallible judges of physical reality. At most, we are infallible judges-in a limited range of circumstances-of our own thoughts and sensations, and of the meanings of the things we say. Hence, we are bound to accept that the properties really possessed by the physical objects we perceive cannot depend upon the properties we think they possess, the properties they seem to us to possess or the terms in which we choose to describe them. For example, if a grassy bank really does have one gross colour rather than another, its gross colour cannot depend on the system of gross colour concepts that we use, or on the gross colours we perceive.

If this moderate view is correct, it is precisely the claims about the relativity of colours which would impugn their reality if they

${ }^{21}$ H. Minkowski, 'Space and time', repr. in A. Einstein et al., The Principle of Relativity (New York: Dover, n.d.; orig. ed. 1923), p. 75. 
were true that turn out to be false. The chromatic colour of a light source is relative to the inertial frame in which it is observed; and in many cases the colour of an object is relative to the atmosphere or light in which it is observed. But gross colours are not relative to systems of concepts or to observers. The thought that they are is a confused way of registering the fact that our system of gross colour concepts is anthropocentric, i.e. that the colours it defines are gross colours relative to trichromatic human beings.

This anthropocentric system of gross colour concepts provides us with a way of identifying gross colours that reflects the characteristics of our visual system, and thereby of the environment in which it evolved, as plainly as the $\mathrm{BC} / \mathrm{AD}$ reference frame for dates reflects the recent history of mankind. The significance of the gross colours we perceive is therefore very local. All but a tiny part of the cosmos is indifferent to colours in general; and since the class of animals that perceives the same gross colours as a normal trichromatic human being is tiny by comparison with the class of sighted animals, the significance of these particular colours is more limited still. For example, if a bull is enraged by a matador's cape, the explanation cannot be that the cape is red, because a bull cannot literally see red.

However, the contrast between local and global is not the same as the contrast between illusion and reality. Like our visual system itself, our system of colour concepts has evolved to perform a variety of related tasks, in a variety of related circumstances. Naturally, these tasks and circumstances are human tasks and human circumstances. Of course, the same is true of all of the concepts that we use, including the most rarified parts of mathematics. But some tasks are more limited in scope and more provincial than some others. My local midwife is as competent as the Minister of Health, but her expertise is more specific and the domain in which she works is smaller. Something similar could be said about colours and shapes. Neither has a greater claim to reality, but one exerts its influence on a much larger stage. ${ }^{22}$

11. Philosophers have claimed that colours are relative to observers, to languages, to systems of concepts, or to 'the human perceptual standpoint'-sometimes without taking much care to distinguish

${ }^{22}$ Something similar can also be said about the colours of physical objects and their spectral reflectances. It is true that surface colours are caused by reflectances, and that reflectances have a broader explanatory competence than colours; but it does not follow that colours are less real. Equally, the shapes of planets are caused by mechanical forces and the shapes of crystals are caused by electrical forces; but it does not follow that the shapes are less real than the forces. 


\section{John Hyman}

between these claims. And they have commonly inferred that colours are less than fully real, by comparison with shapes or intervals in time. In general terms, these arguments depend on a failure to appreciate the significance of the fact that colours cannot be identified except in the context of a frame of reference; on a failure to distinguish with sufficient care between systems of concepts and the facts or truths they are used to express; and on a tendency to slide between a number of distinctions: between reality and illusion, between absolute and relative, between natural and conventional, and between global and local explanatory force.

The truth that lies behind these errors is that our colour concepts are anthropocentric. This is the fact that, seen through a distorting lens, seems to diminish the reality of the colours we perceive. Thus, suppose we confine ourselves, for simplicity, to a single dimension of variation in colour. As we have seen, there is a striking contrast between the constant rate of change in wavelength from one end of the visible spectrum to the other, and the broad bands of approximately homogeneous colour we perceive. Wavelengths of light do not vary continuously: the ancient motto natura non facit saltum is not true. But the imperceptible jumps that nature makes progress across the boundaries between gross colours at an even rate; and our perception of the gross colours in the visible spectrum is explained by the unequal salience-that is, salience to us-of equal intervals along her path. It follows that there is no independent sanction for our system of gross colour concepts-independent, that is, of ourselves. In Plato's phrase, our colour concepts do not 'carve nature at the joints' —or rather, they do, but this is human nature, and not the nature of the visible objects we perceive. ${ }^{23}$

This train of thought seems to me to be correct, and Plato's phrase provides an apt way of saying where it leads. The mistake is to infer that colours are less real than shapes or intervals in time. For Plato's metaphor is not about reality and illusion: it is about explanatory force. The fact it records, in this particular case, is that our system of gross colour concepts can be used to explain the behaviour of human beings, and of other animals that perceive the same gross colours as we do; but that it cannot be used to explain the behaviour of the coloured objects we perceive. It is hard to exaggerate the significance of this point in the theory of colours; but it is easy to misunderstand it. ${ }^{24}$

The Queen's College, Oxford

${ }^{23}$ Phaedrus, 265d-266a.

${ }^{24}$ I examine its significance in J. Hyman, The Objective Eye: colour, form and reality in the theory of art (Chicago, Ill.: Univ. of Chicago Press, 2006), ch.1. 